\title{
IUE Observations of Faint Comets
}

\author{
H. A. WEAVER AND P. D. FELDMAN \\ Physics Department, The Johns Hopkins University, Baltimore, Maryland 21218
}

M. C. FESTOU ${ }^{1}$

Space Physics Research Laboratory, University of Michigan, Ann Arbor, Michigan 48109

M. F. A'HEARN

Astronomy Program, University of Maryland, College Park, Maryland 20742

AND

H. U. KELLER

Max-Planck-Institute für Aeronomie, Katlenburg-Lindau, Federal Republic of Germany

Received April 23, 1981; revised August 24, 1981

\begin{abstract}
Ultraviolet spectra of seven comets taken with the same instrument are presented. Comets P/Encke (1980), P/Tuttle (1980 h), P/Stephan-Oterma (1980 g), and Meier (1980 q) were observed during November-December 1980 with the International Ultraviolet Explorer (IUE) satellite observatory, while comets P/Borrelly (1980 i) and Panther (1980 u) were observed with IUE on 6 March 1981. The spectra of these comets are compared with those of comet Bradfield (1979 X), studied extensively earlier in 1980 with IUE, as well as with each other. In order to simplify the interpretation of the data and to minimize the dependence upon a specific model, the spectra are compared at approximately the same value of heliocentric distance whenever possible. Effects due to heliocentric velocity, geocentric distance, and optical depth are also discussed. All of the cometary spectra are remarkably similar, which suggests that these comets may have a common composition and origin.
\end{abstract}

\section{INTRODUCTION}

The capability of the International Ultraviolet Explorer (IUE) satellite observatory to obtain useful ultraviolet spectra of comets with visual magnitude as faint as 10 has enabled us to observe comets $\mathrm{P} /$ Encke (1980), P/Tuttle $(1980$ h), P/StephanOterma (1980 g), and Meier (1980 q) during November-December 1980 and comets P/Borrelly (1980 i) and Panther (1980 u) on 6 March 1981. The spectra of these comets

Paper presented at IAU Colloquium 61, "Comets: Gases, Ices, Grains, and Plasma,' Tucson, Arizona, March 11-14, 1981.

${ }^{1}$ On leave from Service d'Aéronomie du CNRS, Verrières-le-Buisson, France. are compared with those of comet Bradfield (1979 X), studied extensively earlier in 1980 with IUE, as well as with each other. (In the following text, all letter and numerical designations for these comets will be dropped.) However, simple comparisons of cometary spectra are sometimes misleading, as the relative intensities of the observed emissions depend critically upon a variety of physical and observational parameters. By comparing spectra at approximately the same value of heliocentric distance $(r)$, we eliminate most of the problems associated with the heliocentric dependence of the spectra. If, in addition, we take into account effects due to heliocentric velocity $(\stackrel{r}{r})$ and geocentric distance $(\Delta)$, then we can make model-independent 
conclusions concerning the "intrinsic" similarities and differences in these comets.

\section{SPECTRA}

The IUE covers the entire spectral range from 1150 to $3400 \AA$ by employing two separate spectrographs. For all of the observations discussed here, each spectrograph was used in the low-dispersion mode with the center of brightness of the comet placed in the center of the large aperture. This aperture is $\sim 10 \times 20^{\prime \prime}$ and is roughly elliptical in shape. Used in this manner, the short-wavelength spectrograph has a resolution of $\sim 12 \AA$ and covers the spectral range $1150-1950 \AA$, while the long-wavelength spectrograph covers the range 1900 $3400 \AA$ at a resolution of $\sim 18 \AA$. Further details concerning the instrument have been given by Boggess et al. (1978). All spectra presented here have been processed using the procedure described by Weaver et al. (1981), and all results given refer to averages of emission brightness over a $10^{\prime \prime} \times 15^{\prime \prime}$ area of the center of the aperture.

At first glance, all of the comets we observed with IUE display strikingly similar spectra. Because it was observed over a wide range of heliocentric distance with IUE $(0.71 \mathrm{AU} \leq r \leq 1.55 \mathrm{AU}$; Weaver $e t$ al., 1981) and since it displayed all (except $\mathrm{CO}^{+}$) previously known ultraviolet features (Feldman et al., 1980), comet Bradfield will serve as our "prototype" comet. Comparisons among the "faint" comets can be conveniently made by referring all observations to this standard.

Figure 1 shows a composite spectrum of comet Bradfield in the wavelength range 1150-3200 $\AA$ from four IUE images taken when $r=0.71 \mathrm{AU}$. It is representative of all comet Bradfield spectra in this wavelength range in the sense that no emissions, other than those displayed here, appear in any spectra taken on other dates. Hydrogen $(\mathrm{H})$, oxygen $(\mathrm{O})$, and hydroxyl $(\mathrm{OH})$ are products of water photodissociation, and their ultraviolet emissions are clearly evi- dent in the spectrum of comet Bradfield. Minor species detected by their ultraviolet emission are $\mathrm{C}, \mathrm{S}, \mathrm{C}_{2}, \mathrm{CS}$, and $\mathrm{CO}_{2}{ }^{+}$. Two bands of the $\mathrm{CO}$ fourth positive system were originally identified in the spectrum of comet Bradfield (Feldman et al., 1980), but the observed emissions are weak and their identification is not conclusive. By comparison, Figs. 2-7 show spectra obtained recently for the "faint" comets discussed in this paper. The $\mathrm{OH}(0,0)$ band and the $\mathrm{HI} L \alpha$ line are the strongest emissions detected in all of the cometary spectra. In addition, two other $\mathrm{OH}$ bands, the $(1,0)$ band at $2811 \AA$ and the $(1,1)$ band at $3122 \AA$, are clearly identified in all of these cometary spectra (wavelengths given are band heads).

$\mathrm{H}$ and $\mathrm{OH}$ are the only positively identified species in comets Meier, Stephan-Oterma, and Panther. Spectra taken with the short-wavelength spectrograph are not shown for these three comets since in each case only the $\mathrm{H} L \alpha$ line is seen with the relatively short exposure times used (30 min or less). However, longer exposures (ranging from 60 to $120 \mathrm{~min}$ ) were made with the long-wavelength spectrograph, and some weaker emissions appear above the noise. The $\operatorname{CS}(0,0)$ band at 2576 $\AA$ and $a \mathrm{CO}_{2}{ }^{+}$emission at $2890 \AA$ may be present in the spectra of comets Meier (Fig. 4) and Panther (Fig. 7). The CS(0,0) band may also be present in comet StephanOterma (Fig. 5). An unusual feature in the comet Stephan-Oterma spectrum (Fig. 5) is the unidentified emission at $\sim 2470 \AA$ which seems real. It should be noted that features in the long-wavelength spectrograph falling in the wavelength range below $\sim 2400 \AA$ or above $\sim 3150 \AA$ must be closely scrutinized as to their authenticity due to the increase in noise in this range.

A short-wavelength spectrum is also not shown for comet Borrelly since only $\mathrm{H} L \alpha$ is present in the 15 -min exposure. However, in the 120 -min exposure in the longwavelength spectrograph (Fig. 6), the $\mathrm{CS}(0,0)$ band is clearly visible, while the less conspicuous feature at $\sim 2430 \AA$ is at 


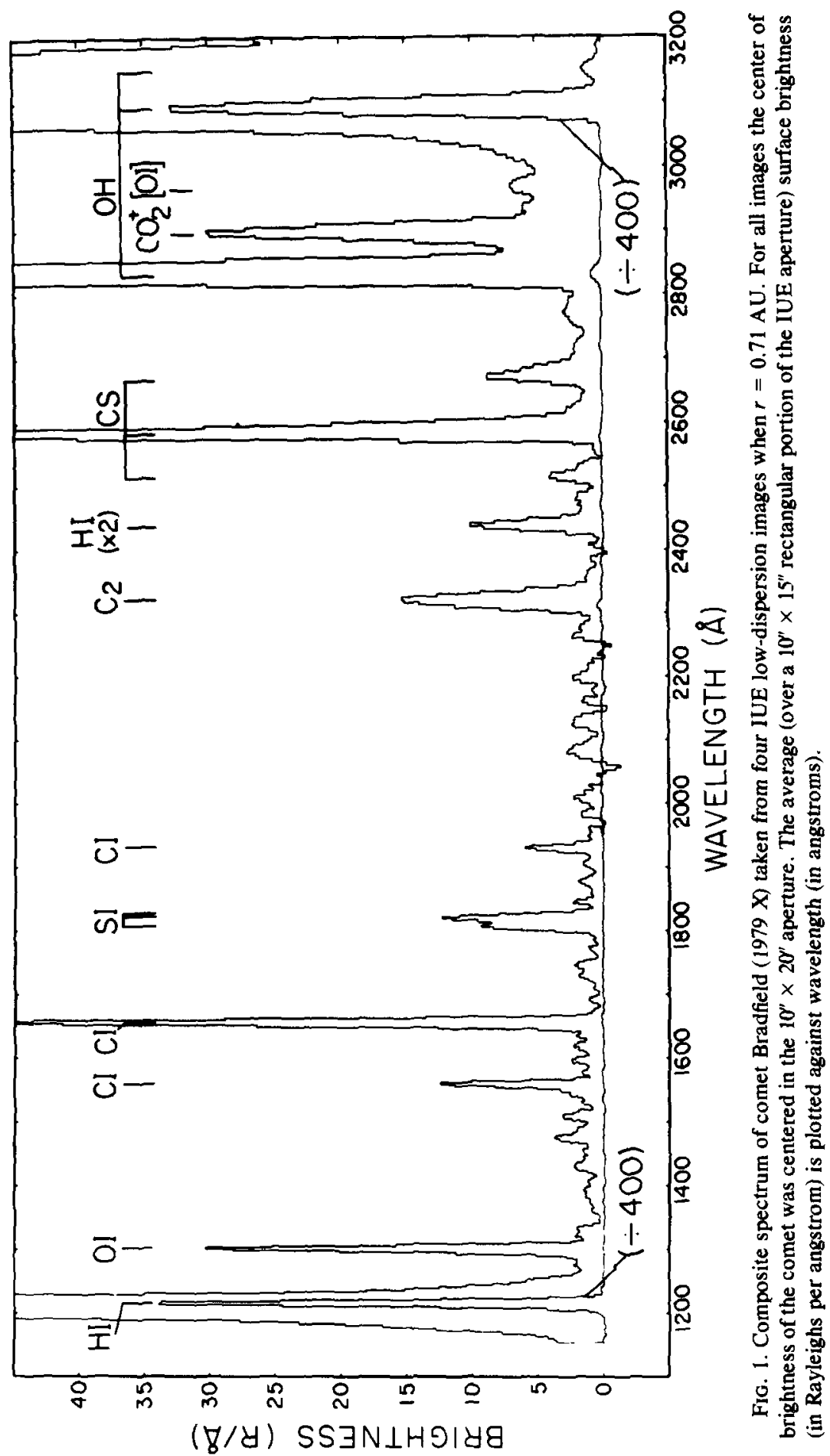




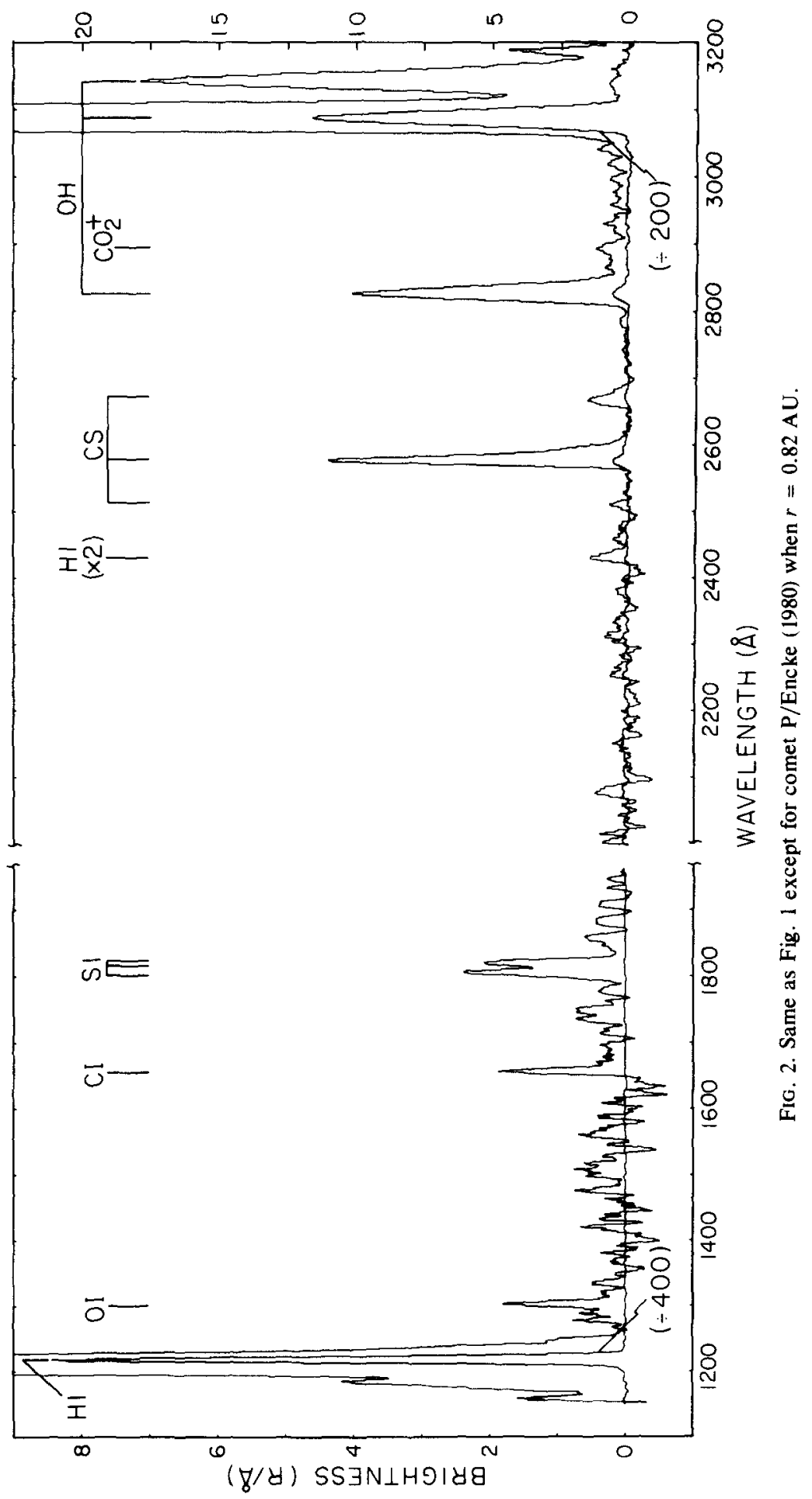




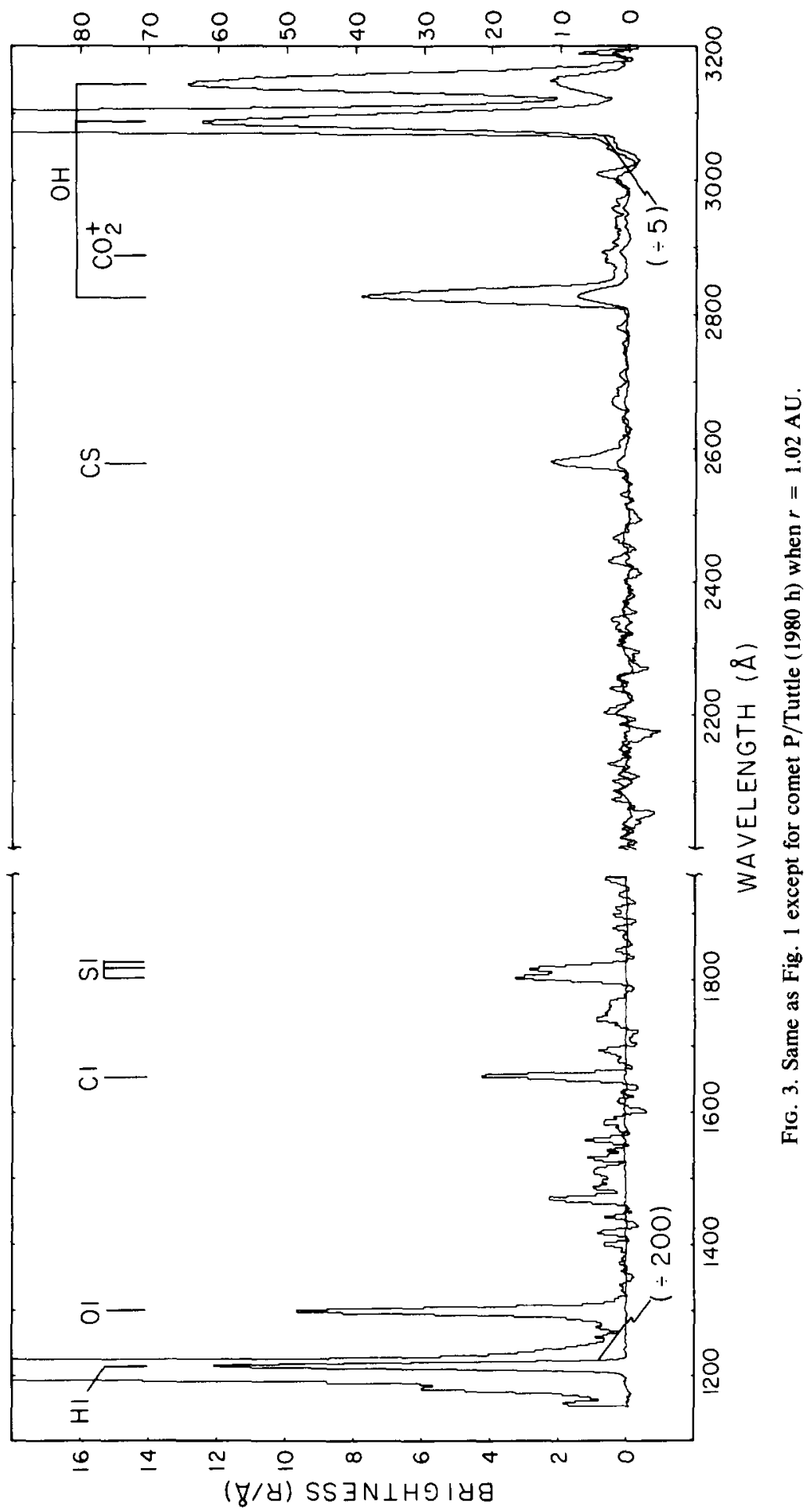




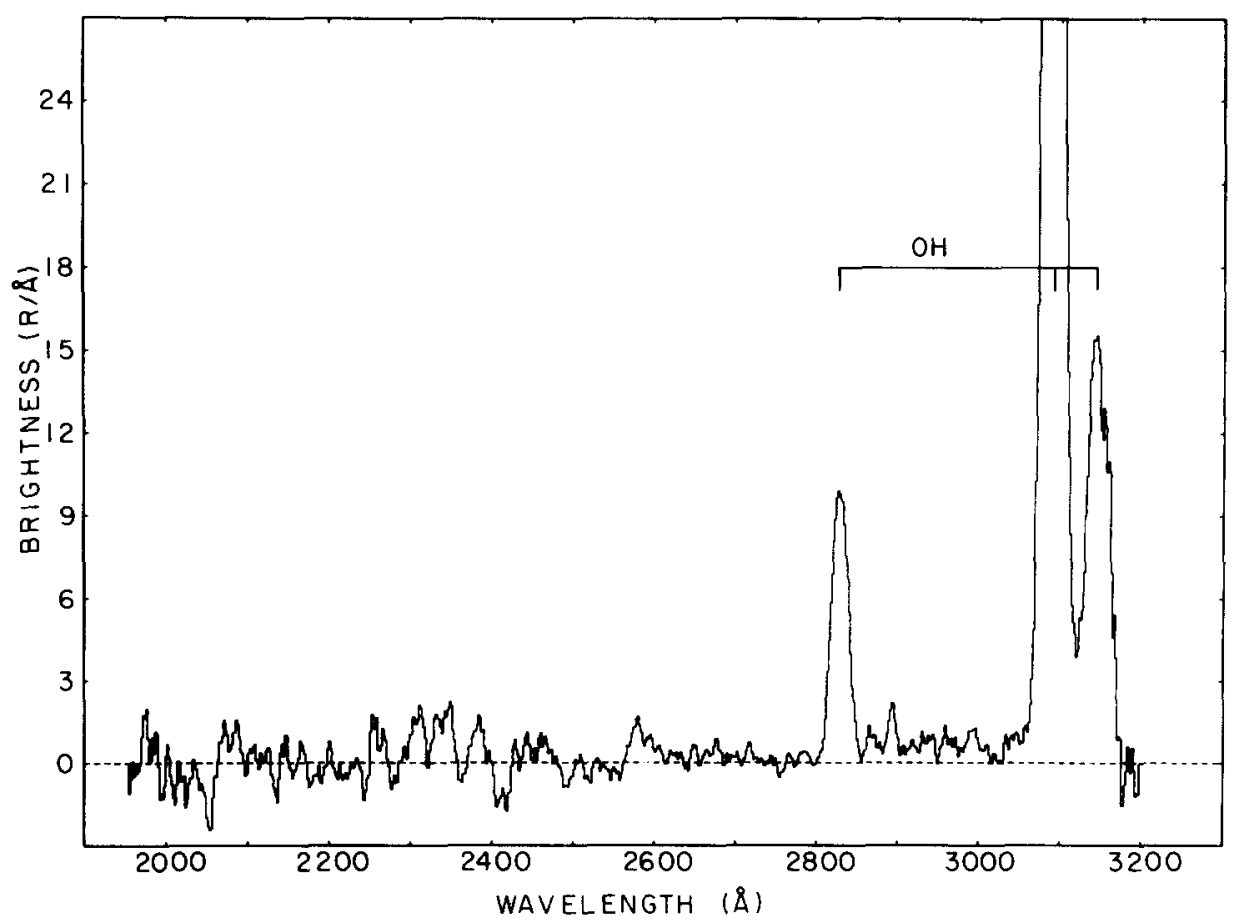

FIG. 4. Spectrum for comet Meier ( 1980 q) from the IUE long-wavelength (LWR) spectrograph when $r=1.52 \mathrm{AU}$. The comet was centered in the aperture and the average surface brightness (over a $10^{\prime \prime} \times$ $15^{\prime \prime}$ portion of the aperture) is plotted against wavelength.

least partially due to second order $\mathrm{HL \alpha}$ signal.

Since we were not able to take long enough exposures on comets Meier, Stephan-Oterma, Panther, and Borrelly to reveal the weaker emissions at the same relative level seen in comet Bradfield, the absence of other atomic or molecular emissions in these comets is not surprising. The long exposures (with both the short- and long-wavelength spectrographs) taken on comets Encke and Tuttle show almost all the features seen in the comet Bradfield spectrum. Besides the $\mathrm{H}$ and $\mathrm{OH}$ emissions, the spectra of comets Encke and Tuttle show emissions from $\mathrm{O}, \mathrm{C}, \mathrm{S}$, and CS (Figs. 2 and 3). It also appears that $\mathrm{CO}_{2}{ }^{+}$ can be distingiushed from the noise, leaving $\mathrm{C}_{2}$ as the only molecule or atom identified in comet Bradfield but not in either comets Encke or Tuttle. Once again, however, this appears to be merely a sensitivity problem since we would not expect to see $\mathrm{C}_{2}$ emission in our spectra if it appears at the same relative level as seen in comet Bradfield (in fact, $C_{2}$ emission could not be positively identified in our spectra of comet Bradfield at $1 \mathrm{AU})$. The $\mathrm{C}_{2}$ molecule is definitely present in these comets as it is identified by its Swan band emission in their visible spectra (A'Hearn, 1981). Furthermore, based on these ground-based measurements and a calculated ratio of Mulliken band to Swan band emission, we verify that the $\mathrm{C}_{2}$ emission should be at or below the detection limit in our ultraviolet spectra. The structure evident in the spectra of comets Encke and Tuttle in the wavelength region $1400-1600 \AA$ is almost certainly instrumental noise, although, as noted earlier for comet Bradfield, this is a region where $\mathrm{CO}$ and $\mathrm{C}$ might contribute weak emissions.

Finally, strong continuum emission ap- 


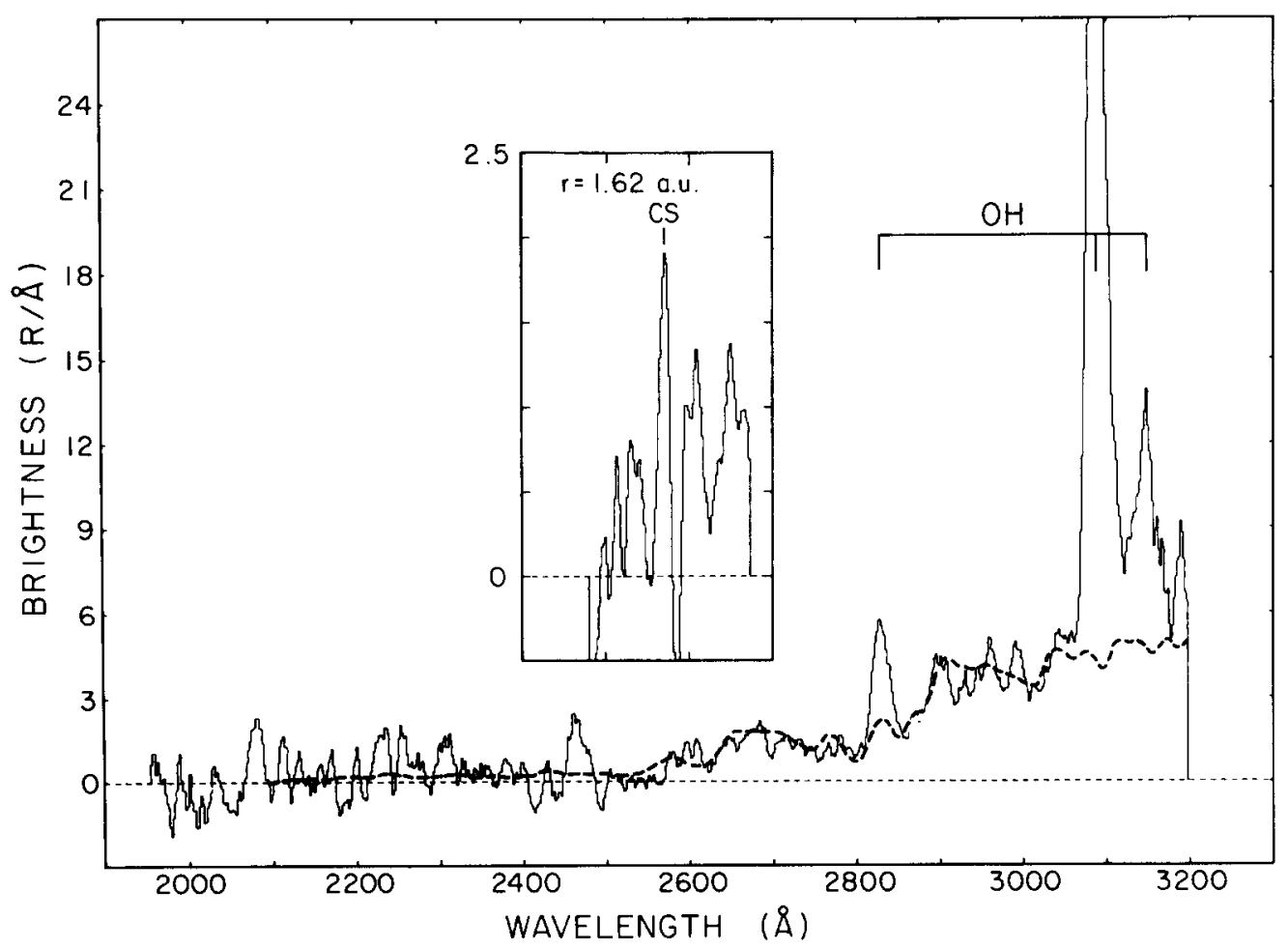

FIG. 5. Same as Fig. 4 except for comet P/Stephan-Oterma when $r=1.58$ AU. Note the strong continuum emission indicative of a high dust to gas ratio for this comet. For comparison purposes, the solar spectrum has been plotted on top of the cometary spectrum. Also shown is the region around $2600 \AA$ taken from another LWR image of comet Stephan-Oterma when $r=1.62$ AU. Since the continuum is weaker relative to the gas emission in this image, emission from the $\operatorname{CS}(0,0)$ band at 2576 $\AA$ is more evident.

pears in the range $2900-3000 \AA$ in the spectra of comets Stephan-Oterma, Panther, and Borrelly which is indicative of a higher dust-to-gas ratio for these comets. The complicated structure evident in this wavelength region is a combination of noise and features which are characteristic of a reflected solar spectrum. For these comets, cometary emission, if present, might be difficult to separate from the solar features.

\section{WATER PRODUCTION RATES}

A Haser model (Festou, 1981a) is used to relate the observed $\mathrm{OH}(0,0)$ band brightness to the water production rate. This model assumes that $\mathrm{H}_{2} \mathrm{O}$ flows radially outward from the nucleus into the coma where it is subsequently photodissociated, primar- ily $(\sim 90 \%)$ into $\mathrm{OH}$ and $\mathrm{H}$. We also assume that water is the only source of $\mathrm{OH}$. Under these circumstances, it has been shown (Festou, 1981a) that the Haser model accurately predicts the $\mathrm{OH}$ column density profile and the water production rate (to within $\sim 20 \%$ ). The principal uncertainty in this procedure is not the inaccuracy of the model but the uncertainty in the values of the model input parameters. These values are chosen to be the same for all the comets studied here. Since the $\mathrm{OH}$ scalelength is known to be a function of the comet's heliocentric velocity (Jackson, 1980), this assumption may introduce some error in the derived water production rates. However, the exact dependence is not known, and our choice of a single $\mathrm{OH}$ scalelength for all 


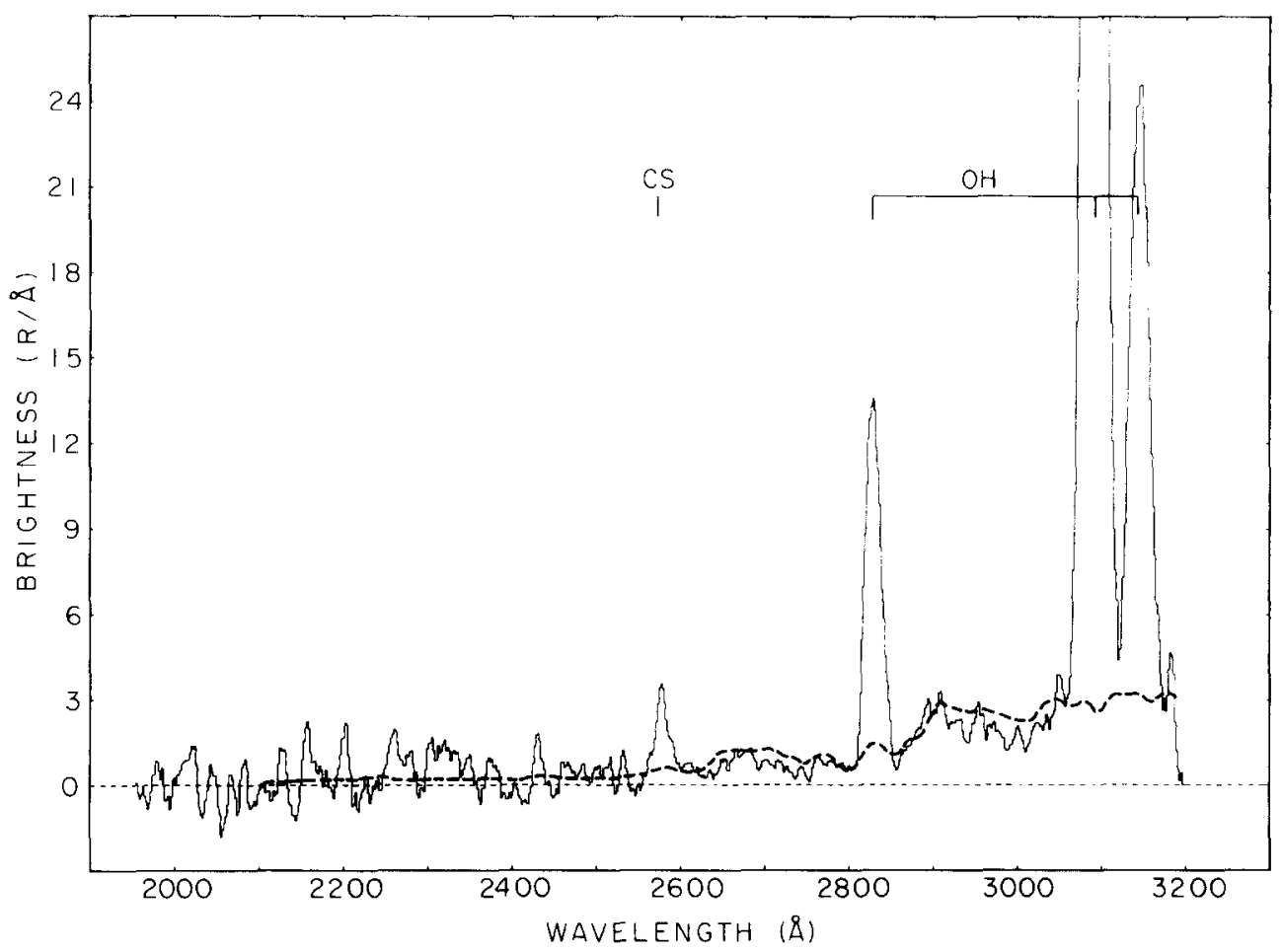

FIG. 6. Same as Fig. 4 except for comet P/Borrelly (1980i) when $r=1.33$ AU. Continuum emission is present and the solar spectrum is plotted on top of the cometary spectrum.

the comets is simply for the sake of convenience. The value $\gamma_{\mathrm{OH}}(1 \mathrm{AU})=5.75 \times 10^{4}$ $\mathrm{km}$ gives a good fit to the comet Bradfield data (Weaver et al., 1981), and this value is adopted here. The $\mathrm{H}_{2} \mathrm{O}$ scalelength and $\mathrm{OH}$ velocity used are $\gamma_{\mathrm{H}_{2} \mathrm{O}}(1 \mathrm{AU})=8.2 \times 10^{4}$ $\mathrm{km}$ and $v_{\mathrm{OH}}=1.15 \mathrm{~km} \mathrm{sec}^{-1}$, respectively (Festou, 1981b).

Table I gives the measured $\mathrm{OH}(0,0)$ band brightness $\left(B_{\mathrm{OH}}\right)$ and the water production rate $\left(Q_{\mathrm{H}_{2} \mathrm{O}}\right)$ derived from it for each of the comets studied. The $\mathrm{OH}$ brightness and water production rate for comet Bradfield at $r$ $=1.7 \mathrm{AU}$ have been extrapolated from the curves given in Fig. 4 by Weaver et al. (1981). The table is divided into sections according to the heliocentric distance $(r)$ of the comet. Other observational parameters listed in Table I are the observation dates, the geocentric distance $(\Delta)$, and the radial component of the heliocentric velocity $(\dot{r})$ for each comet. The excitation factor, or " $g$ factor", for the $\mathrm{OH}$ emission is strongly dependent upon heliocentric velocity (Schleicher and A'Hearn, 1981), and its value at $1 \mathrm{AU}$ is given in Table $\mathrm{I}$ for each comet. We also derive average $\mathrm{OH}$ column densities within a $10^{\prime \prime} \times 15^{\prime \prime}$ aperture (the $\mathrm{OH}(0,0)$ band emission is optically thin) for each comet and these are given in Table I.

It is apparent from Table I that comet Encke is significantly less active than the other comets. However, there is some question as to whether or not P/Encke is significantly more active now than it was during its 1970 apparition. Bertaux et al. (1973), using $\mathrm{H} L \alpha$ isophotes obtained from OGO-5 photometer data to derive a hydrogen production rate for comet Encke, found a water production rate (assuming $Q_{\mathrm{H}_{2} \mathrm{O}}=$ $Q_{\mathrm{H}}$, since Bertaux et al. observed essentially only one of the two hydrogen components) approximately a factor of 5 less than that reported here. They admit, though, 


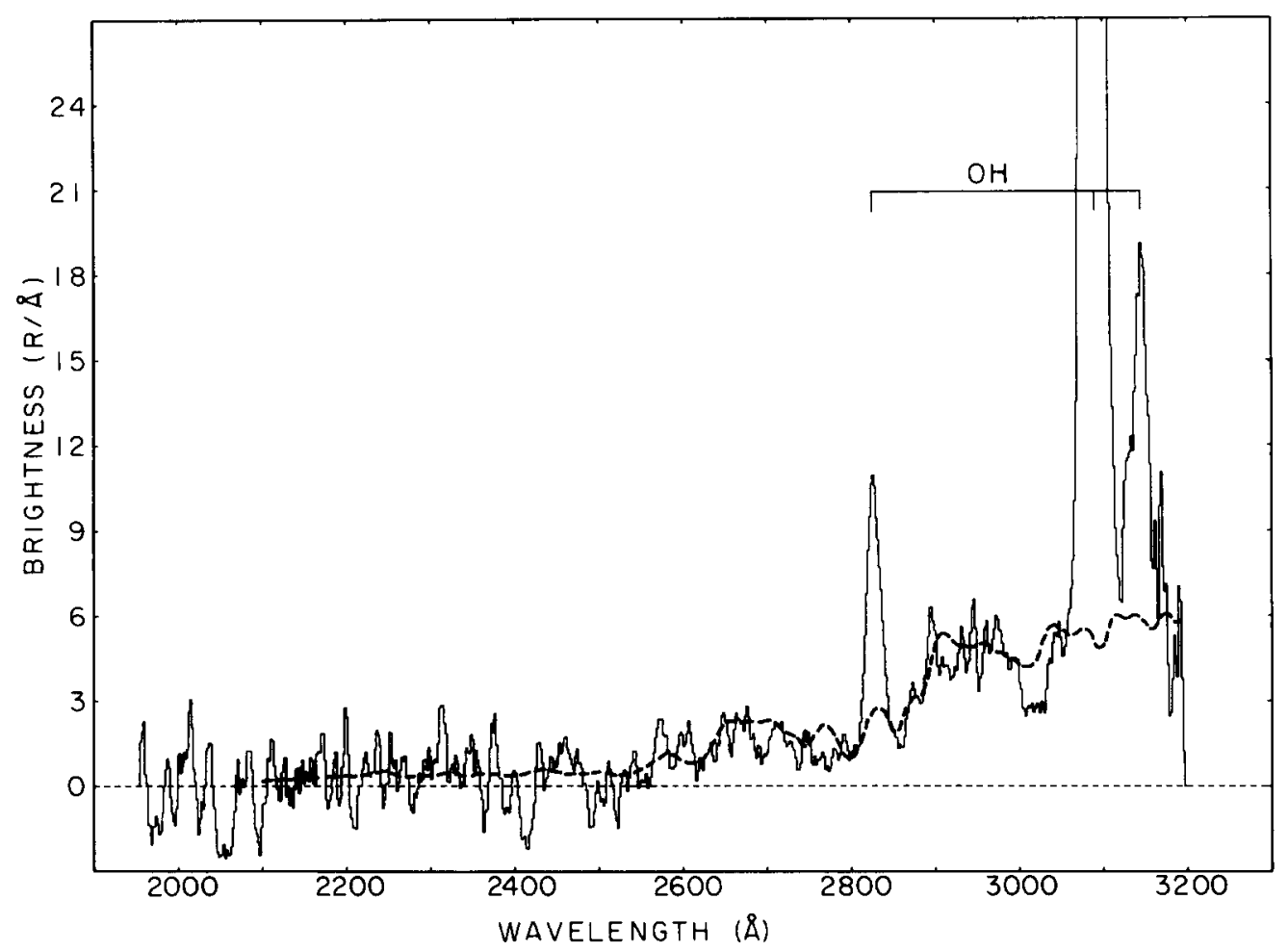

FIG. 7. Same as Fig. 4 except for comet Panther (1980 u) when $r=1.73$ AU. Strong continuum emission is present and the solar spectrum is plotted on top of the cometary spectrum.

that their data were not of sufficient quality to allow a detailed comparison with their model. Since estimates of the visual magnitude (at the same heliocentric distance and reduced to unit geocentric distance) of comet Encke during the 1970 and 1980 apparitions show differences of no more than one magnitude, it is unlikely that the gas production rate was more than a factor of 2 or 3 higher during the 1980 apparition compared to 1970.

The effect of a velocity-dependent $g$ factor is clearly illustrated by comparing the observed brightnesses to the derived production rates for comets Bradfield and Tuttle. Although the $\mathrm{OH}(0,0)$ band brightness for comet Bradfield is about a factor of 4 greater than that for comet Tuttle, the comets have nearly identical water production rates, both being about a factor of 6 larger than that for comet Encke. Finally, an important point to note is the relatively high activity shown by comets Borrelly, Meier, Stephan-Oterma, and Panther despite their large distance from the Sun. In fact, comet Meier at $r=1.52 \mathrm{AU}$ and comet Panther at $r=1.73 \mathrm{AU}$ have larger water production rates than comets Bradfield and Tuttle at $r=1 \mathrm{AU}$.

\section{OXYGEN AND HYDROGEN}

Due to the long exposure times required, the oxygen triplet at $1304 \AA$ was observed only in comets Encke, Bradfield, and Tuttle, and these brightnesses are recorded in Table II. Comparison of oxygen brightnesses from comet to comet requires caution as the excitation factor for oxygen emission is even more sensitive to heliocentric velocity (Feldman et al., 1976) than the $\mathrm{OH} g$ factor. This effect is illustrated by the data in Table II. While comets Bradfield and Tuttle have virtually identical water production rates and $\mathrm{H} L \alpha$ intensities, the 


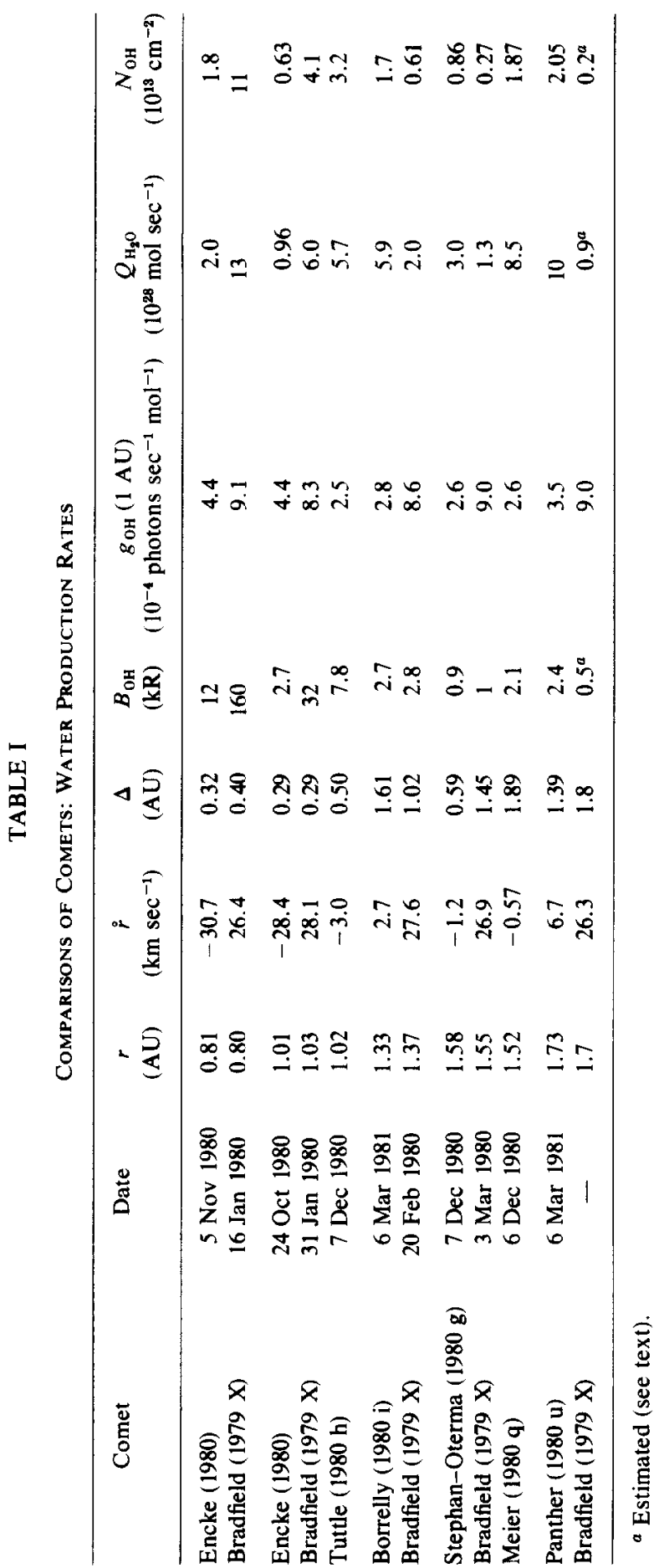


TABLE II

COMPARISONS OF COMETS: HYDROGEN AND OXYGEN

\begin{tabular}{lcccccccc}
\hline \multicolumn{1}{c}{ Comet } & Date & $\begin{array}{c}r \\
(\mathrm{AU})\end{array}$ & $\begin{array}{c}\dot{r} \\
(\mathrm{~km} \mathrm{sec})\end{array}$ & $\begin{array}{c}\Delta \\
(\mathrm{AU})\end{array}$ & $\begin{array}{c}B_{\mathrm{HI}} \\
(\mathrm{kR})\end{array}$ & $\begin{array}{c}N_{\mathrm{H}} \\
\left(10^{13} \mathrm{~cm}^{-2}\right)\end{array}$ & $\begin{array}{c}B_{0 I} \\
(\mathrm{R})\end{array}$ & $\begin{array}{c}N_{\mathrm{O}} \\
\left(10^{13} \mathrm{~cm}^{-2}\right)\end{array}$ \\
\hline Encke (1980) & 5 Nov 1980 & 0.81 & -30.7 & 0.32 & 28.1 & 0.70 & 15 & 0.40 \\
Bradfield (1979 X) & 16 Jan 1980 & 0.80 & 26.4 & 0.40 & 93 & 3.2 & 105 & 2.5 \\
Encke (1980) & 24 Oct 1980 & 1.01 & -28.4 & 0.29 & 6.6 & 0.25 & - & - \\
Bradfield (1979 X) & 31 Jan 1980 & 1.03 & 28.1 & 0.29 & 33.7 & 1.5 & $25^{a}$ & $0.8^{a}$ \\
Tuttle (1980 h) & 7 Dec 1980 & 1.02 & -3.0 & 0.50 & 24.7 & 1.1 & 125 & 1.2 \\
Borrelly (1980 i) & 6 Mar 1981 & 1.33 & 2.7 & 1.61 & 7.1 & 0.48 & - & - \\
Bradfield (1979 X) & 20 Feb 1980 & 1.37 & 27.6 & 1.02 & 3.6 & 0.25 & - & - \\
Stephan-Oterma (1980 g) & 7 Dec 1980 & 1.58 & -1.2 & 0.59 & 2.8 & 0.26 & - & - \\
Bradfield (1979 X) & 3 Mar 1980 & 1.55 & 26.9 & 1.45 & 1.5 & 0.13 & - & - \\
Meier (1980 q) & 6 Dec 1980 & 1.52 & -0.6 & 1.89 & 6.2 & 0.55 & - & - \\
Panther (1980 u) & 6 Mar 1981 & 1.73 & 6.7 & 1.39 & 3.9 & 0.45 & - & - \\
Bradfield (1979 X) & - & 1.7 & 26.3 & 1.8 & 0.6 & 0.06 & - & - \\
\hline
\end{tabular}

${ }^{a}$ Estimated (see text).

oxygen brightness for comet Tuttle is approximately five times that for comet Bradfield (the estimated $O I$ brightness for comet Bradfield should be quite good as it is an extrapolation of the $\mathrm{O} I$ measurements at $0.71,0.80$, and $0.93 \mathrm{AU}$; even at $r=0.93$ AU comet Bradfield had an $\mathrm{OI}$ brightness of only $45 \mathrm{R}$ ). The reason is that the absorption wavelength for comet Tuttle is approximately at the peak of the solar oxygen line while the absorption for comet Bradfield falls on the steeply sloped wing. Because the relative values of the excitation factors depend critically upon the shape of the solar line, and since this shape is not very well known, it is difficult to compare oxygen production from comet to comet in a precise manner. However, the brightness of the oxygen emission in $\mathrm{P} /$ Tuttle, observed when $\dot{r}$ was small, is consistent with a predominently water source of oxygen in the coma. The oxygen column densities listed in Table II are derived assuming that all oxygen atoms are in the lowest fine-structure level of the ground state, and that the exciting solar flux is $4.0,5.1$, and 18 (units : $10^{9}$ photons $\mathrm{cm}^{-2} \mathrm{sec}^{-1} \AA^{-1}$ ) for comets Encke, Bradfield, and Tuttle, respectively. These solar fluxes were determined using the line integrated flux from Mount et al. (1980) and the line shape from recent Solar Maximum Mission measurements (Woodgate, 1981). Since in all cases the optical depth is not negligible, an approximate radiative transfer formalism (Festou et al., 1979) is used to relate the measured surface brightness to the column density.

On the other hand, the hydrogen $L \alpha$ emission is not as sensitive to heliocentric velocity as the oxygen emission, since the solar $\mathrm{H} L \alpha$ line is much broader (VidalMadjar, 1975) than the solar OI line. The $\mathrm{H} L \alpha$ brightness was measured for all the comets discussed here (except that the comet Bradfield $L \boldsymbol{\alpha}$ brightness at $r=1.7$ $A U$ is an extrapolation), and the values are listed in Table II. As expected for a water source, the $L \alpha$ brightnesses scale approximately as the water production rates derived from the $\mathrm{OH}$ observations. Small deviations from this scaling are expected for three reasons. The different comets were observed at different geocentric distances so that the IUE aperture subtended different fractions of the total coma. Also, the $\mathrm{H}$ excitation factor is slightly dependent upon heliocentric velocity since the solar $\mathrm{H} L a$ line is not strictly a constant over the entire 
wavelength interval within which the different cometary absorptions take place. Finally, the comets were not observed on the same dates (also listed in Table II), so that the absolute value of the $H L \alpha$ flux was probably different for each cometary observation. As variations in the solar $\mathrm{H} L \alpha$ flux of $20-30 \%$ over a solar rotation period are not unusual, we would expect the $\mathrm{H}$ excitation factors to show a similar variation. The comparison of comets Encke and Bradfield at $0.8 \mathrm{AU}$ shows the largest departure from what is expected if $\mathrm{H}_{2} \mathrm{O}$ is the dominant source of $\mathrm{H}$ and $\mathrm{OH}$ in both comets. In this case the $L \alpha$ brightness of comet Bradfield is about a factor of 3 greater than that of comet Encke whereas the ratios of water production rates suggest a factor of approximately 6. However, this discrepancy is almost certainly an optical depth effect, since the discrepancy is greatly reduced when we compare the hydrogen column densities derived from the observations and also shown in Table II. When the $\mathrm{HI}$ emission becomes optically thick, the column densities are calculated in the same manner described above for oxygen (in all cases the exciting solar flux is taken to be $5.14 \times 10^{11}$ photons $\mathrm{cm}^{-2} \sec ^{-1} \AA^{-1}$; see Weaver et al., 1981). Also, it should be pointed out that for an $H L \alpha$ brightness less than about $10 \mathrm{kR}$, some uncertainty in the measured cometary emission is introduced by the subtraction of geocoronal $L \alpha$, although a careful subtraction was made based on observations of the geocorona $2^{\circ}$ away from the comet taken nearly simultaneously.

\section{MINOR SPECIES}

Table III gives the measured $\operatorname{CS}(0,0)$ band intensities and the derived CS column densities, as well as the ratios of derived CS production rates to water production rates. Remember, however, that the CS emission was only marginally detected in comets Meier, Stephan-Oterma, and Panther so that the derived column densities and production rates for these comets are probably upper limits. It is also important to note that although the same excitation factor is used in deriving all $\mathrm{CS}$ production rates. $\left(g_{\mathrm{CS}}(1 \mathrm{AU})=7 \times 10^{-4}\right.$ photons $\mathrm{sec}^{-1} \mathrm{~mol}^{-1}$ from Jackson et al. (1981)), the $\operatorname{CS}(0,0)$ band emission most likely shows a strong dependence with heliocentric velocity similar to that of the $\mathrm{OH}(0,0)$ band emission.

TABLE III

Comparisons of Comets: CS Production Rates

\begin{tabular}{|c|c|c|c|c|c|c|c|}
\hline Comet & Date & $\begin{array}{c}r \\
(\mathrm{AU})\end{array}$ & $\begin{array}{c}\stackrel{\circ}{r} \\
\left(\mathrm{~km} \mathrm{sec}^{-1}\right)\end{array}$ & $\begin{array}{c}\Delta \\
(\mathrm{AU})\end{array}$ & $\begin{array}{c}\boldsymbol{B}_{\mathrm{cs}} \\
(\mathbf{k R})\end{array}$ & $\begin{array}{c}Q_{\mathrm{CS}} / Q_{\mathrm{H}_{2} \mathrm{O}} \\
\left(10^{-4}\right)\end{array}$ & $\begin{array}{c}N_{\mathrm{cs}} \\
\left(10^{12} \mathrm{~cm}^{-2}\right)\end{array}$ \\
\hline \multirow[t]{4}{*}{ Bradfield (1979 X) } & $10 \operatorname{Jan} 1980$ & 0.71 & 24.0 & 0.62 & 2.2 & 5 & 1.6 \\
\hline & $16 \operatorname{Jan} 1980$ & 0.80 & 26.4 & 0.40 & 1.2 & 4 & 1.1 \\
\hline & $24 \operatorname{Jan} 1980$ & 0.93 & 27.8 & 0.20 & 0.64 & 2 & 0.79 \\
\hline & 7 Feb 1980 & 1.15 & 28.2 & 0.54 & 0.09 & 3 & 0.17 \\
\hline \multirow[t]{4}{*}{ Encke (1980) } & 5 Nov 1980 & 0.81 & -30.7 & 0.32 & 0.67 & 11 & 0.63 \\
\hline & 4 Nov 1980 & 0.83 & -30.5 & 0.31 & 0.61 & 11 & 0.60 \\
\hline & 3 Nov 1980 & 0.84 & -30.3 & 0.30 & 0.35 & 8 & 0.35 \\
\hline & 24 Oct 1980 & 1.01 & -28.4 & 0.29 & 0.08 & 4 & 0.12 \\
\hline Tuttle $(1980 \mathrm{~h})$ & 7 Dec 1980 & 1.02 & -3.0 & 0.50 & 0.30 & 4 & 0.45 \\
\hline Borrelly (1980 i) & $6 \operatorname{Mar} 1981$ & 1.33 & 2.7 & 1.61 & 0.07 & 5 & 0.18 \\
\hline Meier (1980 q) & 6 Dec 1980 & 1.52 & -0.57 & 1.89 & 0.05 & $\leq 4$ & $\leq 0.17$ \\
\hline Stephan-Oterma (1980 g) & 7 Dec 1980 & 1.58 & -1.2 & 0.59 & 0.04 & $\leq 3$ & $\leq 0.14$ \\
\hline Panther (1980 u) & 6 Mar 1981 & 1.73 & 6.7 & 1.39 & 0.04 & $\leq 3$ & $\leq 0.17$ \\
\hline
\end{tabular}


Nevertheless, two conclusions may be drawn from the data presented in Table III. First, although CS appears very prominently in many of the uv cometary spectra, it is clearly only a minor constituent of the coma, never comprising more than about $0.1 \%$ of the coma. Also, the data for both comets Bradfield and Encke seem to indicate a heliocentric variation in the CS production rate which is different than that for $\mathrm{H}_{2} \mathrm{O}$ (both comets were observed during periods when $r$, and consequently the $g$ factor, remained roughly constant). This behavior in comet Bradfield has been noted by Jackson et al. (1981).

Measurements of carbon and sulfur emission were obtained only for comets Bradfield, Encke, and Tuttle, and the brightnesses and derived column densities are given in Table IV. The brightnesses for comet Bradfield at $1 \mathrm{AU}$ are estimated from the brightness curves for these emissions and should be fairly reliable (certainly within a factor of 2). The $g$ factor for carbon is relatively insensitive to heliocentric velocity varying by about a factor of 2 in the

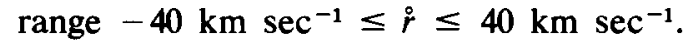
Using the integrated flux in the solar CI (1657 \&) multiplet from Mount et al. (1980) and lineshapes from Solar Maximum Mission measurements (Woodgate, 1981), we derive $\mathrm{g}_{\mathrm{CI}}(1 \mathrm{AU})=3.7,7.0$, and 5.1 (units: $10^{-5}$ photons $\mathrm{sec}^{-1}$ atom ${ }^{-1}$ ) for comets Encke, Bradfield, and Tuttle, respectively. A comparison of the carbon brightness (or column densities) indicate that carbon production in all three comets scales roughly as the water production rate. The sulfur emission observed in these comets is the partially resolved triplet near $1812 \AA$. Like the oxygen emission, the observed transitions are ${ }^{3} \mathrm{P}_{2,1,0}-{ }^{3} \mathrm{~S}$, with the lines excited by the corresponding solar sulfur lines. However, unlike the case for oxygen, the sulfur excitation factor is not as strongly dependent upon heliocentric velocity because of the weakness of the solar feature and the presence of the underlysing solar continuum. We choose $g_{\mathrm{SI}}(1 \mathrm{AU})=6.7 \times$ $10^{-5}$ photons $\sec ^{-1}$ atom ${ }^{-1}$, independent of heliocentric velocity. This corresponds to a continuum solar flux in this region of $1.94 \times$ $10^{10}$ photons $\mathrm{cm}^{-2} \mathrm{sec}^{-1} \AA^{-1}$ (Mount et al., 1980 ). Also unlike oxygen, the sulfur emission is strongly peaked near the nucleus of the comet (the spatial brightness profile is similar to that of the $\operatorname{CS}(0,0)$ band) so that the geocentric distance is an important consideration in comparing sulfur emission among the comets. Keeping all of these effects in mind, it appears that the ratio of sulfur production to water production is approximately the same (within a factor of 2) for the comets at the same heliocentric distance.

\section{CONCLUSION}

Ultraviolet spectra of seven comets are presented in this paper, and all have been observed with the same instruments on IUE. The obvious impression one gets from a first look at these spectra is their remarkable similarity, which suggests that these comets may have a common composition

TABLE IV

Carbon and Sulfur: Comparisons of Comets Encke, Bradfield, and Tuttle

\begin{tabular}{lcccccccc}
\hline \multicolumn{1}{c}{ Comet } & Date & $\begin{array}{c}r \\
(\mathrm{AU})\end{array}$ & $\begin{array}{c}\dot{r} \\
\left(\mathrm{~km} \mathrm{sec}^{-1}\right)\end{array}$ & $\begin{array}{c}\Delta \\
(\mathrm{AU})\end{array}$ & $\begin{array}{c}B_{\mathrm{CI}} \\
(\mathrm{R})\end{array}$ & $\begin{array}{c}N_{\mathrm{c}} \\
\left(10^{12} \mathrm{~cm}^{-2}\right)\end{array}$ & $\begin{array}{c}B_{\text {SI }} \\
(\mathrm{R})\end{array}$ & $\begin{array}{c}N_{S} \\
\left(10^{12} \mathrm{~cm}^{-2}\right)\end{array}$ \\
\hline Encke (1980) & 5 Nov 1980 & 0.81 & -30.7 & 0.32 & 15 & 0.27 & 60 & 0.59 \\
Bradfield (1979 X) & 16 Jan 1980 & 0.80 & 26.4 & 0.40 & 185 & 1.7 & 180 & 1.7 \\
Bradfield (1979 X) & 31 Jan 1980 & 1.03 & 28.1 & 0.29 & $45^{a}$ & $0.68^{a}$ & $90^{a}$ & $1.4^{a}$ \\
Tuttle (1980 h) & 7 Dec 1980 & 1.02 & -3.0 & 0.50 & 50 & 1.0 & 85 & 1.3 \\
\hline
\end{tabular}

\footnotetext{
${ }^{a}$ Estimated (see text).
} 
and origin. The strongest emissions, those of the atoms and molecules most abundant in the comae, are the same in all of the comets observed with IUE to date. The presence of the weaker emissions, those of the minor species, apparently depends solely upon the instrument sensitivity and the comet's heliocentric velocity. The weaker emissions will become visible if we simply take long enough exposures, unless the comet's heliocentric velocity is such that the excitation factor for the emission becomes vanishingly small (e.g., the case of the OI $1304 \AA$ triplet when $r$ is large).

The similarities among the comets become even more remarkable when we examine their spectra in detail. By comparing comets at approximately the same heliocentric distance and taking into account effects due to heliocentric velocity, geocentric distance, and optical depth, we find the observed hydrogen and hydroxyl emissions in all of the comets studied to be consistent with a common water source for these species. Although there is more uncertainty in the analysis of the oxygen emission, it too appears to be derived from water. While CS emission appears fairly prominently in many of the spectra, it is clearly only a minor species in all of these comets, in approximately the same abundance relative to $\mathrm{H}_{2} \mathrm{O}$. Finally, emissions from carbon and sulfur are positively identified in three of the comets (Bradfield, Encke, and Tuttle), also appearing in approximately the same abundance relative to water.

Apparently the only distinguishing characteristic among the comets studied with IUE is the dust to gas ratio, as determined from the relative strength of the continuum emission in the cometary spectra. Comets Stephan-Oterma, Panther, and Borrelly are "dustier" than comets Bradfield, Encke, Tuttle, and Meier.

A final note concerns the gas production rate of comets at large heliocentric distances. Comets Panther and Meier may appear to be "faint," but they are certainly not inactive as indicated by their large wa- ter production rates. Indeed, comets Panther and Meier must be very large since they are producing gas at a rate comparable to that of other comets which are much closer to the Sun and which appear more "bright."

\section{AC KNOWLEDGMENTS}

We gratefully acknowledge the assistance of the IUE Observatory staff in the acquisition and reduction of the satellite date. This research was supported by NASA under Grant NSG 5393 to the Johns Hopkins University and Grant NAG 5-74 to the University of Maryland.

\section{REFERENCES}

A'Hearn, M. F. (1981). Private communication.

A'Hearn, M. F., and Feldman, P. D. (1980). Carbon in comet Bradfield (1979 I). Astrophys. J. Lett. 242, L187-L190.

Bertaux, J. L., Blamont, J. E., and Festou, M. (1973). Interpretation of hydrogen Lyman-alpha observations of comets Bennett and Encke. Astron. Astrophys. 25, 415-430.

Boggess, A., et al. (1978). The IUE spacecraft and instrumentation. Nature 275, 372-377.

Feldman, P. D., Opal, C. B., Meier, R. R, AND Nicolas, K. R. (1976). Far ultraviolet excitation processes in comets. In The Study of Comets (B. Donn et al., Eds.), pp. 773-795. NASA SP-393, Washington, D.C.

Feldman, P. D., et al. (1980). IUE observations of the UV spectrum of comet Bradfield (19791). Nature 286, 132-135.

Festou, M. C. (1981a). The density distribution of neutral compounds in cometary atmospheres. I. Models and equations. Astron. Astrophys. 95, 6979.

Festou, M. C. (1981b). The density distribution of neutral compounds in cometary atmospheres. II. Production rate and lifetime of $\mathrm{OH}$ radicals in comet Kobayashi-Berger-Milon (1975 IX). Astron. Astrophys. 96, 52-57.

Festou, M., Jenkins, E. B., Keller, H. U., Barker, E. S., BertauX, J. L., Drake, J. F., AND UPSON, W. L., II (1979). Lyman-alpha observations of comet Kobayashi-Berger-Milon (1975 IX) with Copernicus. Astrophys. J. 232, 318-328.

JACKSON, W. M. (1980). The lifetime of the $\mathrm{OH}$ radical in comets at $1 \mathrm{AU}$. Icarus 41, 147-152.

Jackson, W. M., Halpern, J., Feldman, P. D., and RAHE, J. (1981). Production of CS and $S$ in comet Bradfield (1979 X). Submitted for publication.

Mount, G. H., Rottman, G. J., And Timothy, J. G. (1980). The solar spectral irradiance $1200-2550 \AA$ at Solar Maximum. J. Geophys. Res. 85, 4271-4274.

SCHLEICHER, D. G., AND A'HEARN, M. F. (1980). 
Fluorescence of $\mathrm{OH}$ in comets. Bull. Amer. Astron. Soc. 12, 462; details in preparation.

VIDAL-MADJAR, A. (1975). Evolution of the solar Lyman alpha flux during four consecutive years. Solar Phys. 40, 69-86.
Weaver, H. A., Feldman, P. D., Festou, M. C., AND A'HearN, M. F. (1981). Water production models for comet Bradfield (1979 X). Astrophys. J., 251, in press.

Woodgate, B. E. (1981). Private communication. 